\title{
Active Damping based on the Capacitor Voltage Positive-Feedback for Grid-Connected Power Converters with LCL filter
}

\author{
Javier Samanes and Eugenio Gubia \\ Department of Electrical, Electronic and Communications Engineering, \\ Insitute of Smart Cities, Public University of Navarre \\ Campus de Arrosadia, 31006 \\ Pamplona, Spain \\ Phone: +34 948169738 \\ Email: javier.samanes@unavarra.es \\ URL: http://www.unavarra.es
}

\section{Acknowledgments}

This work has been supported by the Spanish State Research Agency (AEI) and FEDER-UE under grant DPI2016-80641-R.

\section{Keywords}

$\ll$ Active damping $\gg$, «Converter control $\gg, \ll$ Frequency-Domain Analysis $》, ~ «$ Voltage Source Converter $(\mathrm{VSC}) \gg$.

\begin{abstract}
The capacitor voltage positive-feedback is a widely extended active damping strategy. It can effectively damp the $L C L$ output filter resonant poles for low ratios of resonance to sampling frequencies. However, the existing delays in the control loop limit the applicability of the capacitor-voltage positive-feedback. For high ratios of resonance to sampling frequencies, it becomes ineffective and can even destabilize the system. This limitation is overcome in this paper by adjusting the delay in the feedback path. With the delay adjustment, a robust damping can be achieved if the delays are properly considered, including the filters, and the grid impedance variations are taken into account. Simulation results validate the proposed active damping strategy.
\end{abstract}

\section{Introduction}

$L C L$ filters are widely used in grid-connected renewable energy systems, such as photovoltaic, wind and energy storage systems [1]. These filters are an appropriate solution, in terms of size and cost, to comply with the limitations imposed by the grid-codes to the injected current harmonic content. However, the resonance of the $L C L$ filter impedances and their interaction with the grid impedance, arise stability issues. To solve the instability problems, while avoiding additional losses introduced by passive damping solutions [2], active damping strategies have been widely explored in the literature [3].

Among these active damping strategies, the capacitor current proportional feedback emulates a resistance in parallel with the filter capacitor, damping the resonant poles [4, 5]. Alternatively, the capacitor current could be estimated using the capacitor voltage derivative [6,7]. This strategy is equivalent to the capacitor current proportional feedback, so both of them suffer the same problems. The emulated virtual resistance is affected by the existing delays in the control loop, and thus, it becomes a virtual impedance $[4,5,7]$, whose real term (resistive component) can become negative creating a destabilizing effect. Even though this problem was solved in a recent work [7], both strategies have some additional drawbacks. For 
the case of the derivative, in some systems where the filters do not introduce enough attenuation to the switching harmonics, noise amplification problems can exist, while the capacitor current is not normally measured in power converters and the required additional sensors increase the system cost. A different approach to damp the $L C L$ resonant poles is the introduction of filters and lead-lag compensator in the main current control loop $[8,9,10]$. These approaches can effectively stabilize the possible instabilities introduced by the combined effect of the resonance frequency and the control delays, but they often fail to provide enough damping as to avoid grid current oscillations at the resonance frequency, and thus, compromise grid code compliance. One of the most popular active damping strategies is the capacitor voltage positive-feedback (CVPF) $[11,12,13]$. This positive-feedback can damp the $L C L$ filter resonant poles but it has additional benefits. The positive-feedback of this voltage is also used to avoid large transient inrush currents when the converter is connected to the grid and improve the converter response during voltage sags. All these benefits explain its popularity.

The capacitor voltage positive-feedback is also influenced by the existing computational and measurement delays, limiting its applicability to low resonance to sampling frequencies. To overcome this limitation, which becomes specially important in high power converters, a capacitor positive-feedback scheme was proposed in [11], using a proportional action, a first order and a second order derivative, to compensate the delays. With this approach, the resonance is effectively damped, but noise amplification issues may arise, specially due to the use of second order derivatives. As an alternative, [13] proposed a method to compensate the existing delays, which includes a modification of the converter's output voltage update instant. However, this strategy may distort the converter current, as it modifies the switching orders in the middle of a sampling period. [12] recently studied the influence that the delays in the capacitor voltage positive-feedback path have on the damping provided to the resonant poles. The effects of these delays are accurately characterized, and two stability regions are clearly identified. However, in order to enlarge the stability region they propose a modification of the capacitor voltage sampling instant, which, in some cases, may not be performed in the carrier wave peaks and valleys, something that can increase measurement noise and introduce aliasing.

In this work, a robust active damping based on the capacitor voltage positive-feedback is proposed that overcomes the previously presented problems related to noise. Moreover, it is robust to variations in the grid impedance, something that becomes specially critical in weak grids, as these variations have a greater influence on the $L C L$ resonant poles [7]. A multi-loop approach is introduced: a capacitor voltage positive-feedback loop will be used for low frequencies to improve the converter response against gridtransients, while an additional capacitor positive-feedback loop will be used for higher frequencies, covering the whole the range of possible resonance frequencies. This additional loop includes a delay adjustment in order to robustly damp the filter resonant poles that does not imply a modification of the voltage sampling instants.

\section{Analysis of the Capacitor Voltage Positive-Feedback}

In Fig. 1 (a) a three phase power converter connected to the grid is represented. It has an an $L C L$ output filter, where $L_{c}$ is the converter inductance and $i_{\text {conv }}$ its current, $L_{g t}$ is the sum of the grid and the step-up transformer leakage inductance, $i_{g}$ the grid current, $C$ is the filter capacitance and $v_{c}$ its voltage. For this system, as a first step, the stabilizing effect of the capacitor voltage positive-feedback is considered for an ideal control loop without delays. Later, the effects on the CVPF of the existing delays in a real system are analyzed, taking into account the computation and filters.

\section{Ideal implementation of the capacitor voltage positive-feedback}

If an ideal control loop, without delays, is considered, the control diagram represented in Fig. 1 (b) is obtained. In this diagram, the converter output current is controlled in the stationary reference frame $\alpha \beta$. The system is consequently modeled in the same reference frame, where a decoupled model between both axis is obtained, being the reason why only the $\alpha$ control loop has been represented. In this diagram $G_{v v}(s)$ is the plant transfer function correlating the voltage applied by the converter in $\alpha, V_{c o n v, \alpha}(s)$ and the capacitor voltage in the same axis, $V_{c, \alpha}(s) . G_{i v}(s)$ is the transfer function correlating the converter 

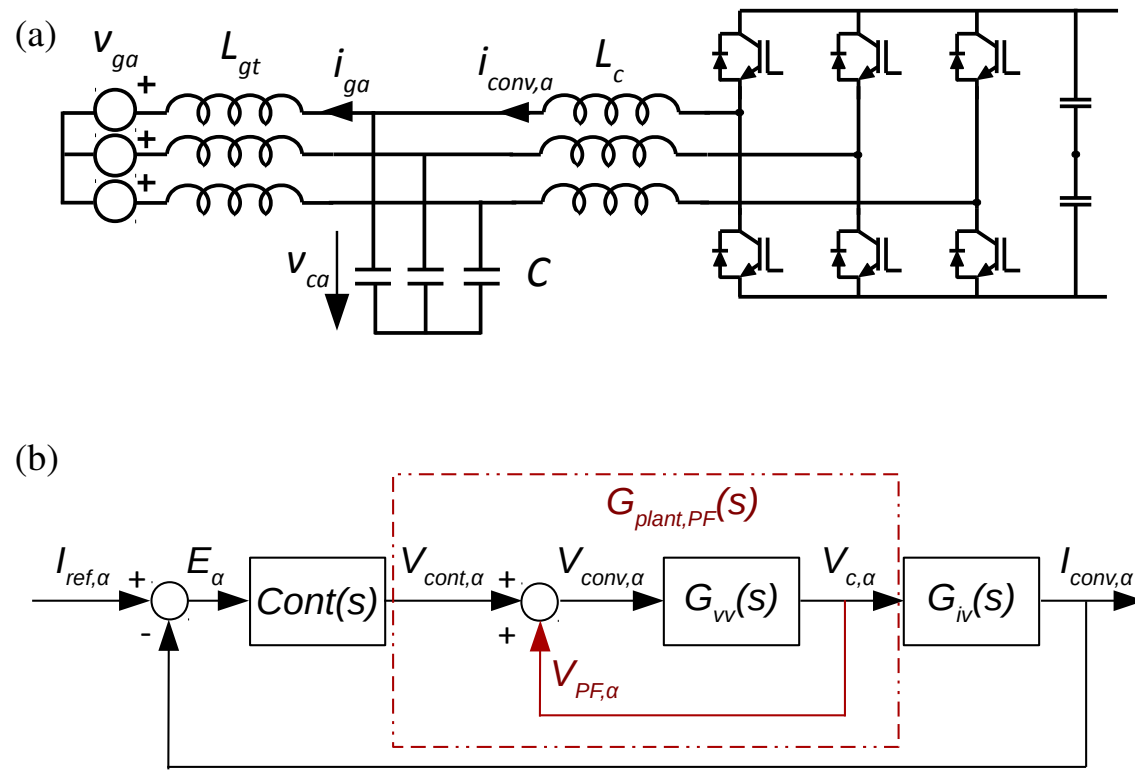

Fig. 1: Three-phase grid-connected power converter with an $L C L$ filter (a) and block diagram of the ideal converter current control loop including the capacitor voltage positive-feedback (b).

current, $I_{c o n v, \alpha}(s)$ and $V_{c, \alpha}(s)$. If the parasitic resistances are neglected, these transfer functions are equal to:

$$
\begin{array}{r}
G_{v v}(s)=\frac{L_{g t}}{C L_{g t} L_{c} s^{2}+\left(L_{g t}+L_{c}\right)} \\
G_{i v}(s)=\frac{C L_{g t} s^{2}+1}{s L_{g t}}
\end{array}
$$

The capacitor voltage positive-feedback is represented in Fig. 1 (b) in red. With this unitary positivefeedback, the plant to be controlled, which is equal to $G_{\text {plant }, P F}(s) \times G_{i v}(s)$, is highly simplified and becomes equal to the converter inductor admittance:

$$
G_{p l a n t, P F}(s) \times G_{i v}(s)=\frac{I_{c o n v, \alpha}(s)}{V_{c o n t, \alpha}(s)}=\frac{G_{v v}(s)}{1-G_{v v}(s)} G_{i v}(s)=\frac{1}{L_{c} s}
$$

As it can be seen, in Eq. 3 the influence of $L_{g t}$ and $C$ are eliminated, canceling the resonant poles. The resulting plant becomes a pure inductor equal to the converter inductance value. This parameter is perfectly known, and the influence of uncertainties in the system are avoided. The last element to be defined in Fig. 1 (a) is Cont $(s)$, which is the proportional resonant controller commonly used in this reference frame to achieve zero tracking error.

\section{Real implementation of the capacitor voltage positive feedback}

In a real application, the existing delays due to the digital implementation of the control loops and measurement filters strongly influence the capacitor voltage positive-feedback. In the block diagram in Fig. 2, three new transfer functions have been added to the ideal current control loop to represent its real implementation. $G_{c o n v}(s)$ models the computational delay and the delay in the application of the reference voltage calculated by the controller. Normally, the variables are sampled in the peaks and valleys of the carrier wave, what is called asymmetrical sampling, in order to reduce noise in the 


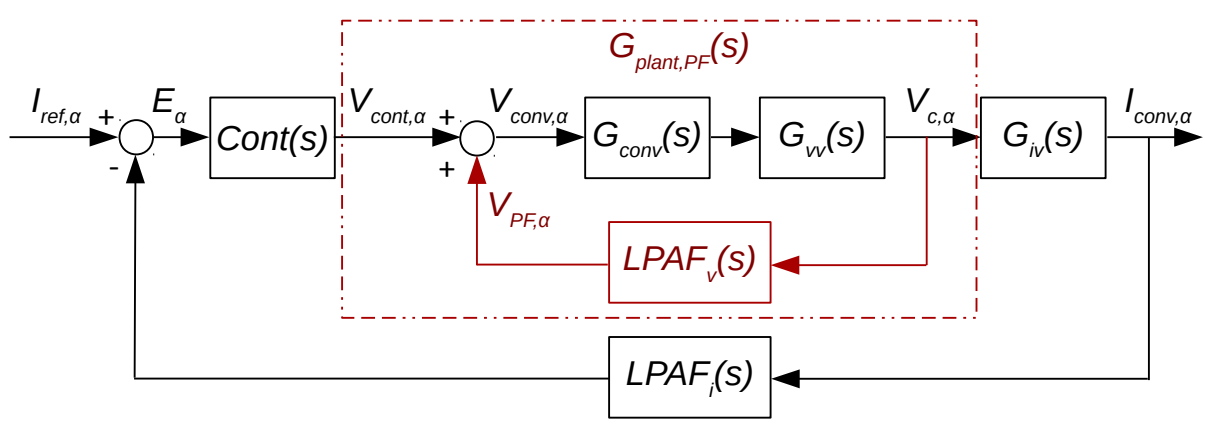

Fig. 2: Block diagram of the converter current control loop including the capacitor voltage positivefeedback.

measurements. This means that the variables are sampled twice per switching period, and the converter output voltage is also updated at the same rate. The exact expression of $G_{c o n v}(s)$ contains pure delays, so, in order to perform computational calculations, it is normally approximated. In this work, the fourth order approximation proposed in [10] is used, as it can effectively characterize the effects of $G_{\text {conv }}(s)$ at high frequencies close to the Nyquist frequency, where the resonance frequency may be located. The other two transfer functions, $L P A F_{v}(s)$ and $L P A F_{i}(s)$, are the first order low pass analog filters used to filter the capacitor voltage and the converter current, respectively. $L P A F_{v}(s)$ has a time constant equal to $\tau_{v}$ and $L P A F_{i}(s)$ equal to $\tau_{i}$.

With these considerations, the expression for the plant to be controlled, $G_{p l a n t, P F}(s) \times G_{i v}(s)$, is obtained:

$$
\begin{aligned}
G_{p l a n t, P F}(s) \times G_{i v}(s) & =\frac{G_{\text {conv }}(s) G_{v v}(s)}{1-G_{\text {conv }}(s) L P A F_{v}(s) G_{v v}(s)} G_{i v}(s) \\
& =\frac{G_{\text {conv }}(s)\left(C L_{g t} s^{2}+1\right)}{s\left(C L_{g t} L_{c} s^{2}+\left(L_{g t}+L_{c}\right)-G_{\text {conv }}(s) L P A F_{v}(s) L_{g t}\right)}
\end{aligned}
$$

The resonance frequency is not canceled, and the damping provided to the resonant poles is strongly dependent on the grid inductance, the filter time constant and the delays introduced by $G_{c o n v}(s)$. It can be seen that in this case, the effectiveness of the capacitor voltage positive-feedback is limited by $G_{c o n v}(s)$ and $L P A F_{v}(s)$.

In Fig. 3 the stability of the CVPF represented in Fig. 2 for the parameters in Table I is analyzed. Fig. 3 (a) shows the Bode diagram for the inner capacitor voltage positive-feedback, from $V_{c o n v, \alpha}(s)$ to $V_{P F, \alpha}(s)$ for three short circuit ratios (SCRs) equal to 1, 40 and 100. Normally, the stability of a negative feedback loop can be determined by counting $\pm n 180^{\circ}$ crossings ( $n$ odd integer) with positive magnitude in the Bode diagram [14]. The loop under study has a positive feedback, for this reason, in this Bode diagram, $-G_{c o n v}(s) G_{v v}(s) L P A F_{v}(s)$ has been represented. It can be seen that at the resonance frequency, a -180 degree crossing is observed for SCRs equal to 40 and 100. For these cases, the combined effect of the $L C L$ resonant poles and the phase delay of $G_{c o n v}(s)$ and $L P A F_{v}(s)$ create a negative crossing with positive magnitude. In this way, for both SCRs the cosed-loop system will have 2 unstable poles. The poles of $G_{p l a n t, P F}(s) \times G_{i v}(s)$ are represented in Fig. 3 (b) for the three different SCRs. It can be seen that for the weakest case under consideration, which implies the lowest resonance frequency, the capacitor voltage positive-feedback is able to damp the resonant poles. If the SCR is increased, meaning that the resonance frequency is also increased, the damping provided is decreased and the capacitor voltage positive-feedback tends to destabilize the system. The destabilizing effect can be correlated with the phase delay introduced in Eq. 4 by $G_{\text {conv }}(j \omega) L P A F_{v}(j \omega)$. If the resonance frequency is denoted by $\omega_{\text {res }}$, the phase $\varphi\left(G_{\text {conv }}\left(j \omega_{\text {res }}\right) L P A F_{v}\left(j \omega_{\text {res }}\right)\right)$ decreases as the SCR increases and the $L C L$ resonant poles move towards higher frequencies. The effect of the increasing phase delay in the damping provided by the capacitor voltage to the resonant poles in Eq. 4 is plotted in Fig. 4 (a). The negative damping 
(a)

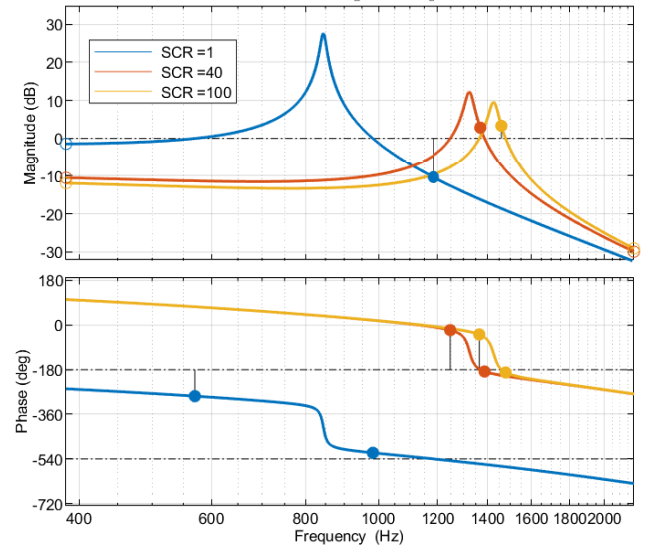

(b)

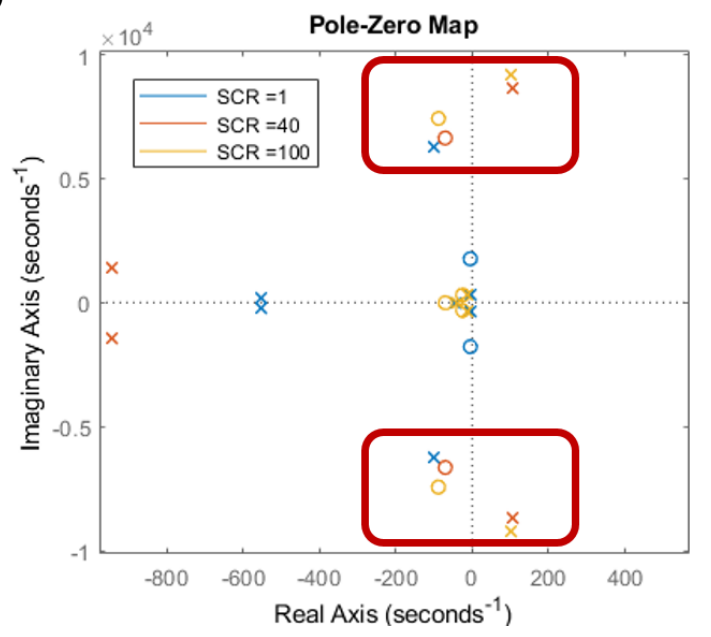

Fig. 3: Bode diagram of open-loop inner CVPF (a) and poles of $G_{p l a n t, P F}(s) \times G_{i v}(s)$ for three different SCRs (b).

represented in this figure indicates that the resonant poles are located in the right half plane (RHP). From Fig. 3 (a) we can conclude that the resonant poles introduce $180^{\circ}$, which create a crossing with positive magnitude if the delay introduced in the capacitor voltage positive-feedback is greater than 180 degrees. Otherwise, if the delay is lower, a crossing is not produced, so the CVPF has a stabilizing effect if

$$
\varphi\left(G_{\text {conv }}\left(j \omega_{\text {res }}\right) L P A F_{v}\left(j \omega_{\text {res }}\right)\right)<-\pi,
$$

otherwise, the CVPF introduces unstable poles in the plant to be controlled. The stability region can be visually identified if the sine of $\varphi\left(G_{\text {conv }}(j \omega) L P A F_{v}(j \omega)\right)$ is represented against frequency, Fig. 4 (b), where the stable region is identified as the red area under the curve. For low ratios of the resonance to sampling frequencies, $F_{\text {samp }}$, the CVPF has a stabilizing effect, while it tends destabilize the system for ratios greater than 0.2. The possible range of resonance frequencies of the $L C L$ output filter is represented by the dashed lines. In this way, the CVPF cannot provide a robust damping for different grid conditions due to the existing delays in a real power converter. To enlarge the stability region, $L P A F_{v}(s)$ could be

(a)

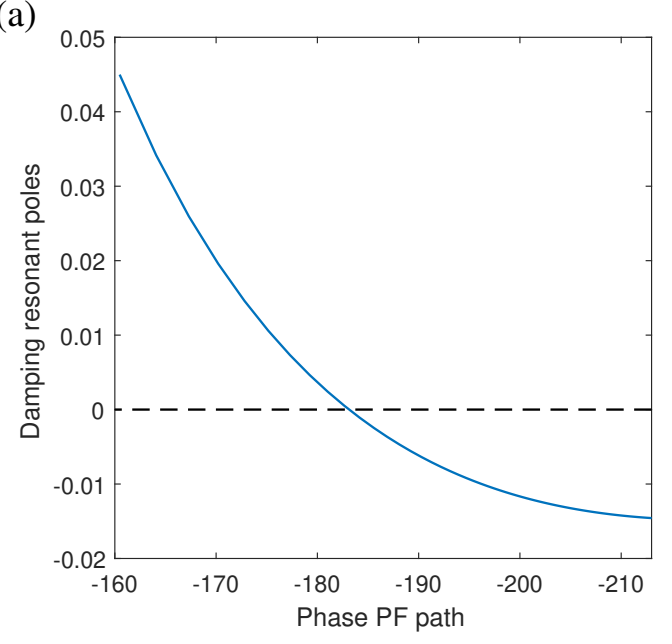

(b)

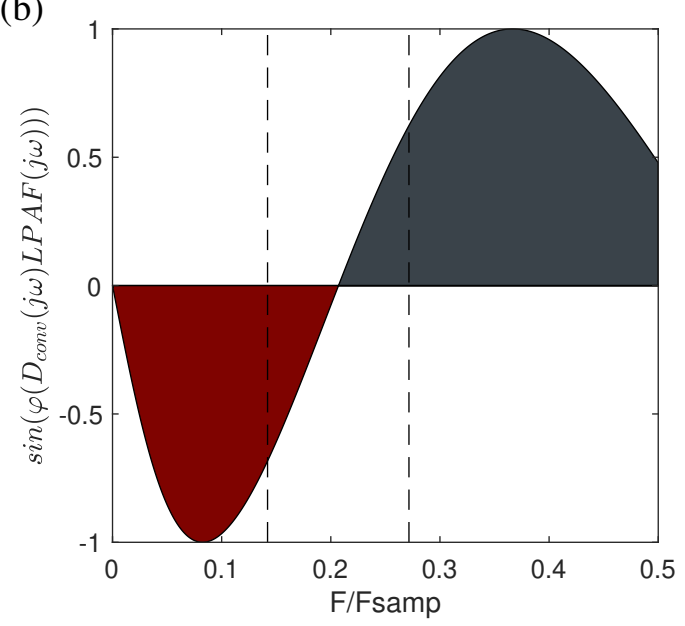

Fig. 4: Evolution of the damping provided to the resonant poles by the capacitor voltage positivefeedback as a function of the delay of $\varphi\left(G_{\operatorname{conv}(j \omega)} L P A F_{v}(j \omega)\right)$ (a) and sine of the phase of $\left(G_{\text {conv }(j \omega)} L P A F_{v}(j \omega)\right)$ against frequency (b). 
Table I: System parameters.

\begin{tabular}{l|c|l|c}
\hline Parameter & Value & Parameter & Value \\
\hline$L_{c}$ & $400 \mu \mathrm{H}$ & $S_{\text {rated }}$ & $500 \mathrm{~kW}$ \\
$L_{\text {transf }}$ & $150 \mu \mathrm{H}$ & $V_{g}$ & $690 \mathrm{~V}$ \\
$C$ & $100 \mu F$ & $F_{S w} / F_{\text {samp }}$ & $2800 / 5600 \mathrm{~Hz}$ \\
$\mathrm{SCR}$ & $1-100$ & $\tau_{L P v}$ & $350 \mu \mathrm{s}$ \\
\hline
\end{tabular}

designed to filter higher frequencies, reducing the delay, but in this case, noise problems can arise, a similar issue that would occur if the capacitor voltage sampling instant is modified to reduce the delay in $G_{c o n v}(s)$ as proposed in the literature. For these reasons, in the next section a new proposal is made for the CVPF in the next section.

\section{Proposed Capacitor-Voltage Positive-Feedback}

Depending on the grid at which the power converter is connected and the delays existing in the feedback path, the CVPF can introduce unstable poles at the resonance frequency. As the delays cannot be well compensated or reduced, a low pass digital filter is added in the already existing feedback path represented in red in Fig. 5, denoted by $\operatorname{LPDF}(s)$ in its equivalent Laplace representation. This filter is a second order Butterworth filter used to eliminate the destabilizing effect of the CVPF for high SCRs. A Butterwoth filter is selected because it offers a good compromise between magnitude flatness and low delay below the cut-off frequency. This filter cut-off frequency is selected to be half of the lowest possible resonance frequency, $F_{\text {res }}$. In this way, the main benefits at low frequencies, related to the improvement of the controller response against grid transients are maintained, while the destabilizing effect at the highest possible resonance frequencies is almost negligible as the magnitude is highly attenuated.

Additionally, as shown in Fig. 5, a second capacitor voltage positive-feedback path, represented in green, is added to damp the $L C L$ resonant poles. This new branch has to guarantee that the new capacitor voltage positive-feedback is only going to actuate in the desired range of frequencies as well as to guarantee the robust damping of the $L C L$ output filter. These goals are achieved by properly adjusting the proposed transfer function $G_{A D}(s)$, which includes the following elements:

1. A filter to limit the range of frequencies where the new CVPF actuates. This filter is a high pass digital filter, denoted by $\operatorname{HPDF}(s)$ in its equivalent Laplace representation. It is a first order filter with a cut-off frequency also selected to be half of the lowest possible resonance frequency, $F_{\text {res }}$, avoiding in this way the interactions with the first CVPF.

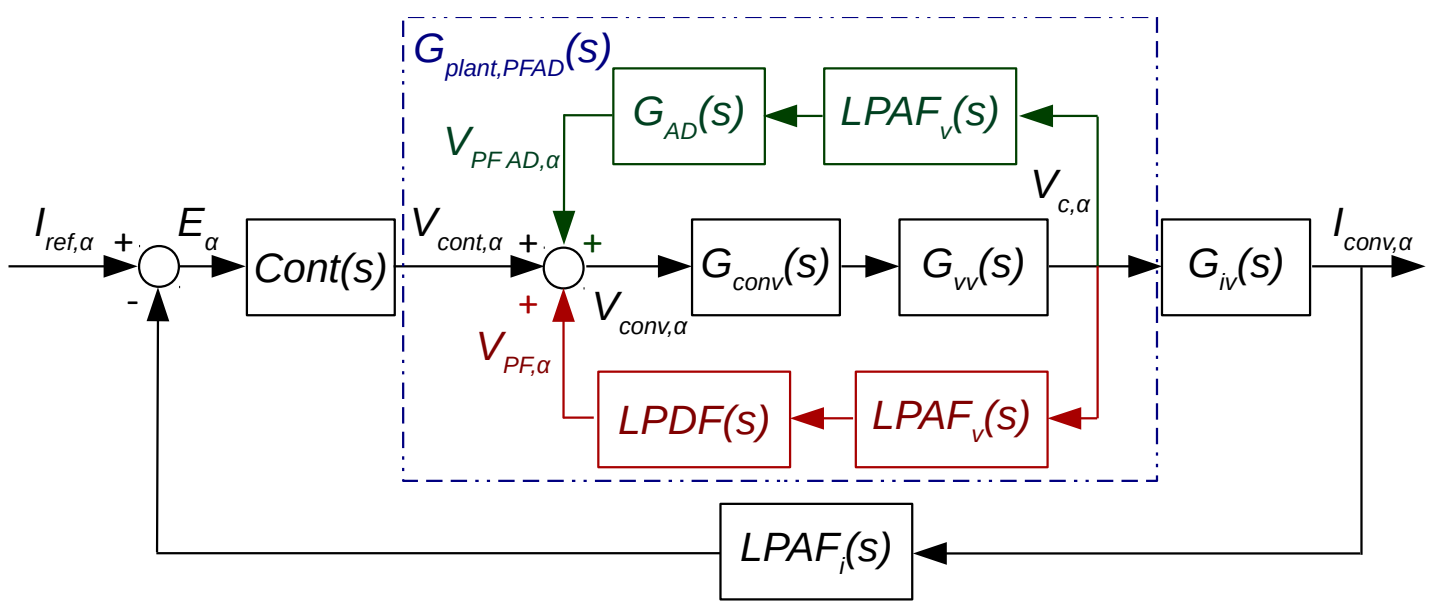

Fig. 5: Block diagram of the converter current control loop including the proposed capacitor voltage positive-feedback to damp the resonance. 
2. An adjustable delay, denoted by $\operatorname{Del}_{A D}(s)$, which is responsible of adjusting the phase within the possible range of resonance frequencies to avoid the destabilizing sign inversion effect described in Section. $\operatorname{Del}_{A D}(s)$ is equal to a pure delay of $y$ sample times, $e^{-y T_{\text {samp }} s}$, where $T_{\text {samp }}$ is the sampling period. With this purpose, the phase delay in the new path is adjusted at the central resonance frequency, defined as the mean of the theoretical highest possible resonance frequency, $F_{r e s_{h}}$, and the lowest possible resonance frequency, $F_{\text {res }}$. The required delay, $y$, is adjusted so that the phase in the $\mathrm{AD}$ feedback path at the central resonance frequency is equal to $\pm n \frac{p i}{2}$ ( $n$ odd integer). In this way, the resonant poles, which introduce 180 degree phase delay, are less likely to produce a $\pm n 180^{\circ}$ crossing ( $n$ odd integer) with positive magnitude at any resonance frequency. The delay can be adjusted by means of the following expression:

$$
\begin{aligned}
& \varphi\left(G_{\text {conv }}\left(j \omega_{\text {cent }}\right) L P A F_{v}\left(j \omega_{\text {cent }}\right) G_{A D}\left(j \omega_{\text {cent }}\right)\right)= \\
& \varphi\left(G_{\text {conv }}\left(j \omega_{\text {cent }}\right) L P A F_{v}\left(j \omega_{\text {cent }}\right) H P D F\left(j \omega_{\text {cent }}\right) e^{-y T_{\text {samp }} j \omega_{\text {cent }}}\right)= \pm n \frac{p i}{2}, n \text { odd integer }
\end{aligned}
$$

The sine term of the phase delay in the new CVPF branch is represented for this adjustment in Fig. 6 (a). It can be seen that for the whole range of possible resonance frequencies the delay is adjusted within -180 and -360 degrees. Compared to Fig. 4 (b), it can be seen that a sign inversion is produced, and thus, the gain $k_{P F A D}$, adjusted in the next step, must be negative.

3. At last, a gain, $k_{P F A D}$ is included. From the analysis performed in the previous step, it must be negative. To determine its value, the Bode diagram in Fig. 6 (b) is carefully examined. In this Bode diagram the CVPF open-loop transfer function in Fig. 5 is represented, which is equal to $-G_{A D}(s) L P A F_{v}(s) G_{v v}(s) G_{c o n v}(s)$. For this initial analysis, $k_{P F A D}$ has been set equal to -1 . The delay adjusted in the previous step, along with the sign inversion in $k_{P F A D}$, guarantee that no $\pm 180^{\circ}$ crossings with positive magnitude occur at resonance frequencies. However, for the weakest grid under consideration, a crossing occurs at $730 \mathrm{~Hz}$. The magnitude plot at the phase-crossover frequency allows to obtain the minimum CVPF gain, $k_{P F A D}$ min, which, in this case, is equal to -0.73 . In this way, $k_{P F A D}$ is set equal to -0.65 avoiding possible instabilities.

The robust damping of the $L C L$ requires $G_{A D}(s)$ to be equal to:
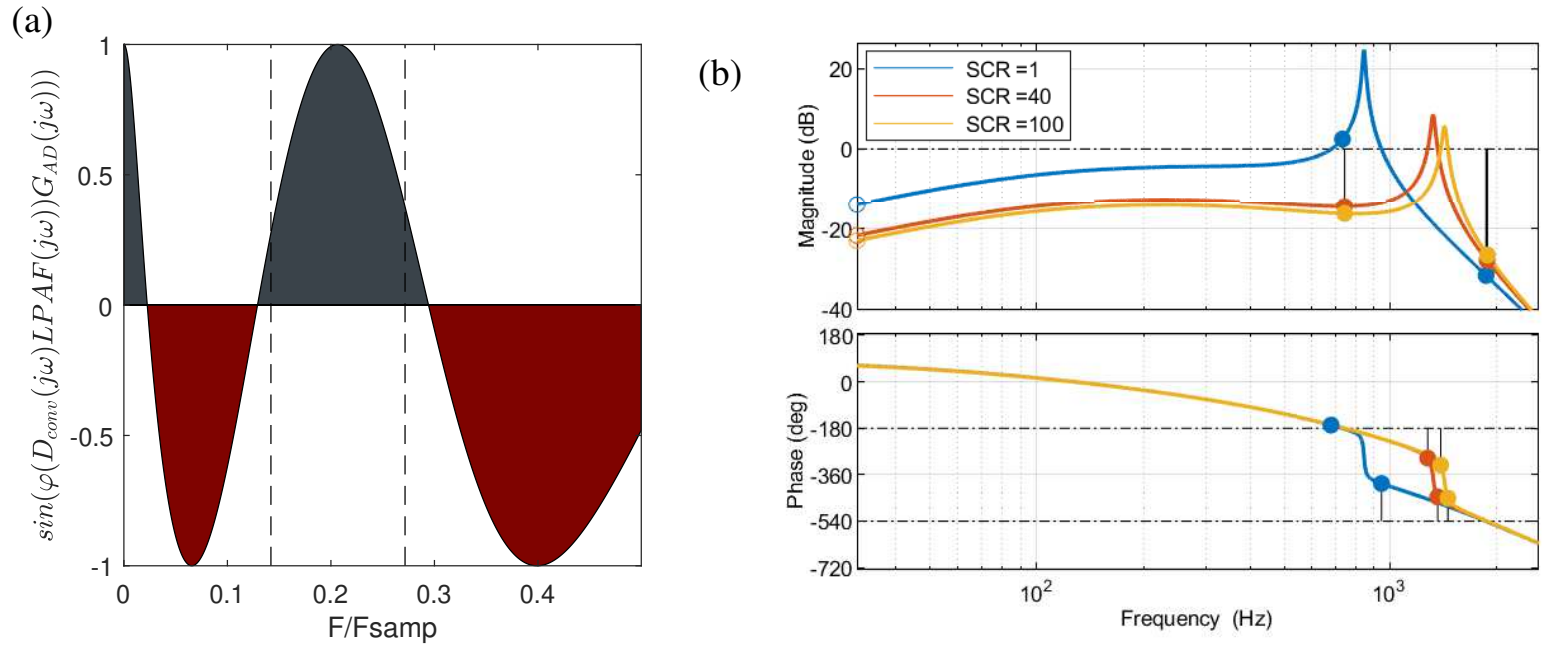

Fig. 6: Sine of the phase in the CVPF against frequency after being adjusted (a) and Bode diagram of the open-loop inner AD feedback (b). 


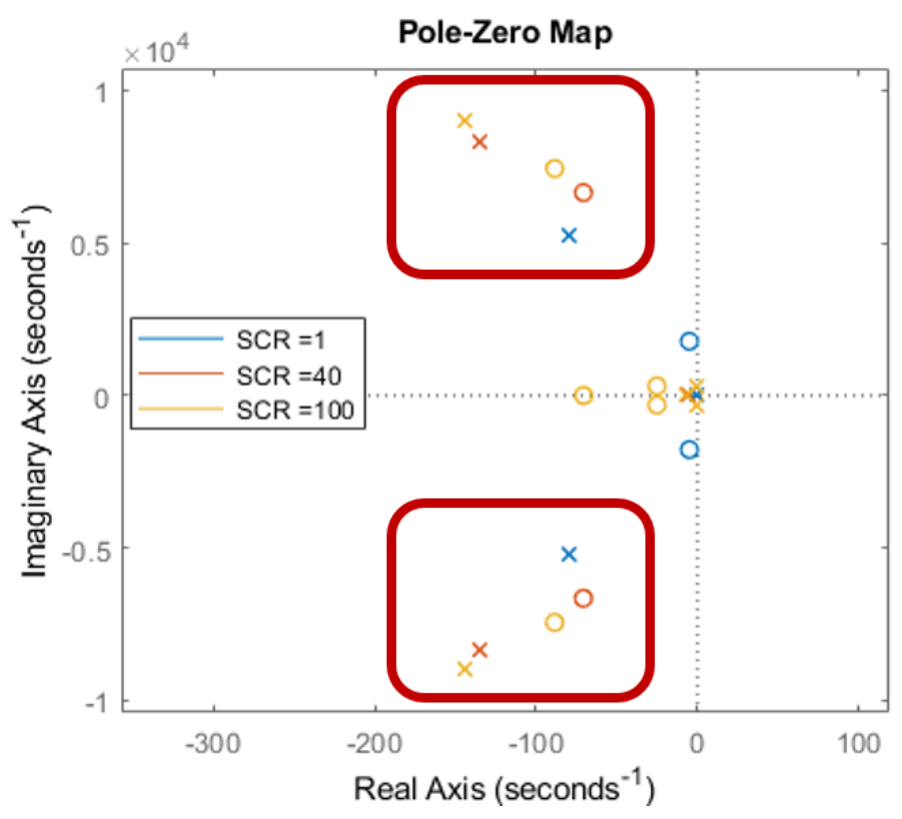

Fig. 7: Poles of $G_{\text {plant }, P F A D}(s) \times G_{i v}(s)$ for three different SCRs.

$$
G_{A D}(s)=k_{P F A D} \operatorname{Del}_{A D}(s) H P D F(s)
$$

Once the AD path is adjusted, the poles of $G_{p l a n t, P F}(s) G_{i v}(s)$, are shown in Fig. 7. It can be seen that the resonant poles of the open loop transfer function to be controlled, denoted by $G_{\text {plant }, \text { PFAD }}(s) G_{i v}(s)$, once that the multiloop proposal is included, it stabilizes the resonant poles for every possible SCR.

\section{Simulation Results}

Simulations are carried out for the $500 \mathrm{~kW}$ power converter of Table I in order to demonstrate the effectiveness of the proposed active damping. The simulations are performed in Matlab using SimPowerSystems.

The active damping strategy has been tested for two different grids at which the power converter can be connected. First, a strong grid with a SCR equal to 100. As it has been theoretically demonstrated, the traditional implementation of the CVPF has a destabilizing effect for this SCR, while it has been verified
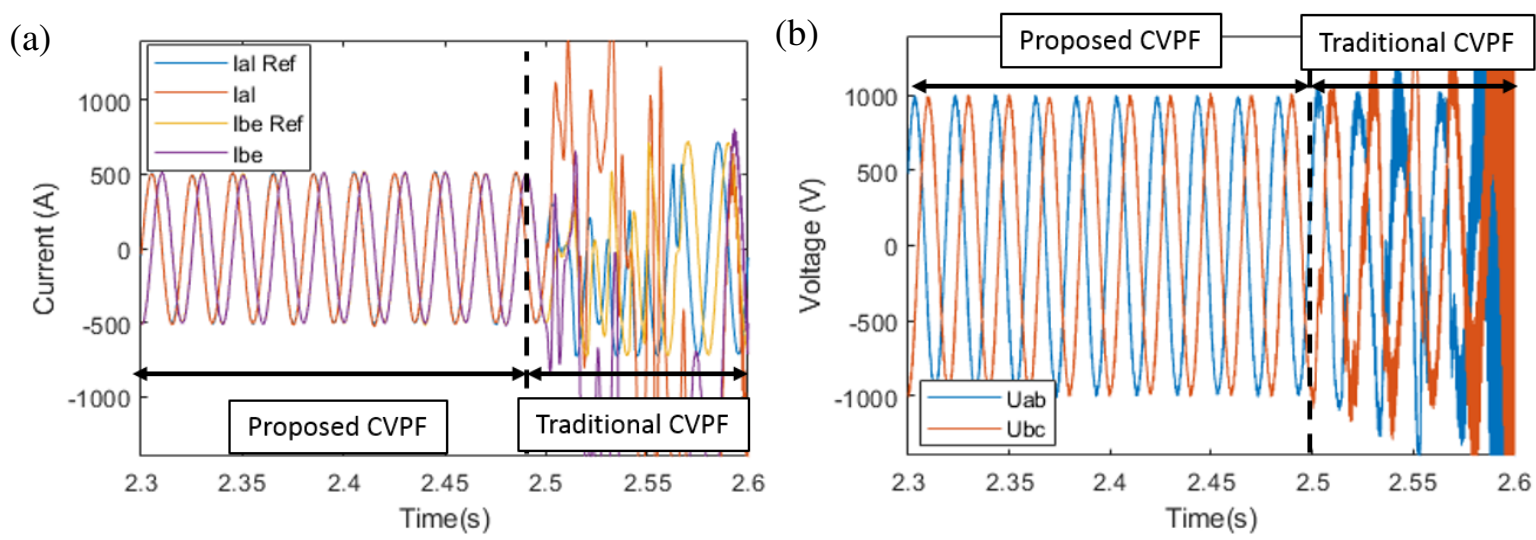

Fig. 8: Grid current waveform (a) and capacitor line voltage (b) for a $\mathrm{SCR}=100$, transitioning from the proposed CVPF to the traditional CVPF. 
(a)

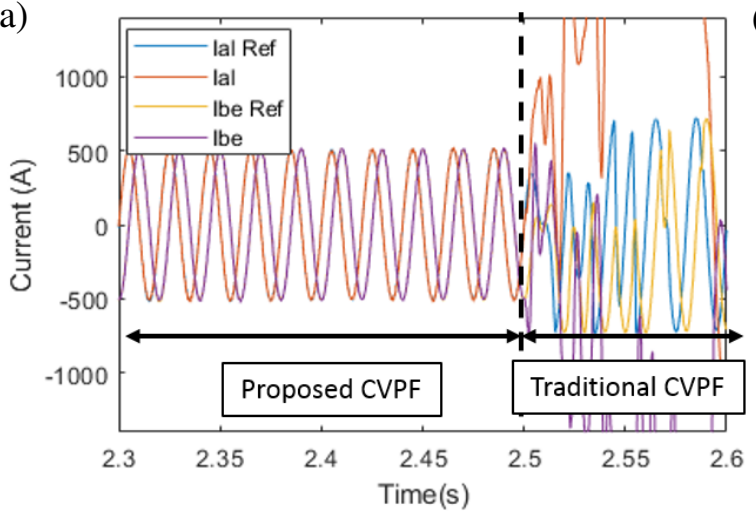

(b)

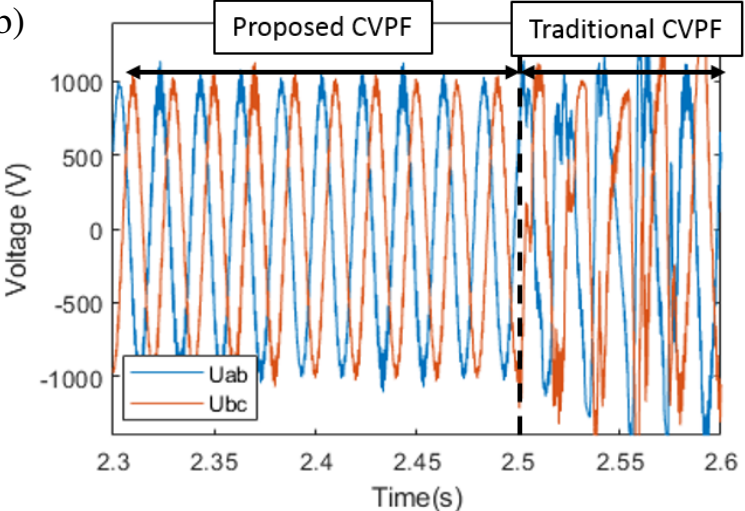

Fig. 9: Grid current waveform (a) and capacitor line voltage (b) for a $\mathrm{SCR}=2$, transitioning from the proposed CVPF to the traditional CVPF.

that with the proposed implementation the system operates in a stable way. This behavior is perfectly correlated to the results obtained from the simulation model. Initially, in Fig. 8 (a) the grid current is shown with the proposed CVPF activated proving that the system is stable, while it becomes unstable as the traditional implementation is used. The same effect can be seen in the line voltages waveforms represented in Fig. 8 (b). Second, a week grid with a SCR=2 is simulated. In this case, both the traditional and the proposed CVPF have a stabilizing effect. However, as it can be concluded from Fig. 9 (a) and (b), the proposed implementation is able to stabilize the system, while the traditional implementation does not provide enough damping and the system becomes unstable.

\section{Conclusion}

In this work an alternative implementation of the capacitor voltage positive feedback is proposed to avoid the instabilities that can occur with its traditional implementation, instability problems that are originated by the delays in the capacitor voltage positive-feedback. The new proposal includes a low bandwidth CVPF to improve the converter response against grid transients, which does not have an influence on the $L C L$ resonant poles. In addition, a different CVPF is included to robustly damp the resonant poles. This new CVPF only actuates within the possible range of resonance frequencies, with an adjustment of the delay and a proportional gain properly selected. A robust damping is achieved for strong and weak grids as it can be concluded from the analysis presented in this work.

\section{References}

[1] IEEE Application Guide for IEEE Std 1547, IEEE Standard for Interconnecting Distributed Resources With Electric Power Systems, IEEE Standard 1547.2-2008.

[2] R. N. Beres, X. Wang, M. Liserre, F. Blaabjerg and C. L. Bak: A Review of Passive Power Filters for ThreePhase Grid-Connected Voltage-Source Converters, IEEE Journal of Emerging and Selected Topics in Power Electronics, Vol 4, number 1, pp. 54-69, 2016.

[3] J. Xu and S. Xie: LCL-resonance damping strategies for grid-connected inverters with LCL filters: a comprehensive review, Journal of Modern Power Systems and Clean Energy, Vol 6, number 2, pp. 292-305, 2018.

[4] D. Pan, X. Ruan, C. Bao, W. Li, X. Wang, Capacitor-current-feedback active damping with reduced computation delay for improving robustness of $L C L$-type grid-connected inverter, IEEE Transactions on Power Electronics, Vol. 29, pp. 34143427, 2014.

[5] S. Parker, B. McGrath, D. Holmes: Regions of active damping control for LCL filters, IEEE Transactions on Industry Applications, Vol. 50, pp. 42432, 2014.

[6] Z. Xin, P. Loh, X. Wang, F. Blaabjerg and Y. Tang: Highly Accurate Derivatives for LCL-Filtered Grid Converter With Capacitor Voltage Active Damping, IEEE Transactions on Power Electronics, Vol. 31, number 5, pp. 3612-3625, 2015.

[7] J. Samanes, and A. Urtasun, E. Gubia and A. Petri: Robust multisampled capacitor voltage active damping for grid-connected power converters, International Journal of Electrical Power \& Energy Systems, Elsevier, Vol 105, pp. 741-752, 2019. 
[8] J. Dannehl, M. Liserre, F. Fuchs: Filter-based active damping of voltage source converters with $L C L$ filter. IEEE Transactions on Industrial Electronics, Vol 58, pp. 36233633, 2011.

[9] J. Wang, Y. Yan, L. Jiang, J. Zou:. Delay-dependent stability of single-loop controlled grid-connected inverters with $L C L$ filters. IEEE Transactions on Power Electronics, Vol 31, pp. 74357, 2016.

[10] J. Samanes, and E. Gubia: Sensorless active damping strategy for parallel interleaved voltage source power converters with LCL filter, 2017 IEEE Applied Power Electronics Conference and Exposition (APEC), pp. 3632-3639, 2017.

[11] W. Li, X. Ruan, D. Pan and X. Wang: Full-Feedforward Schemes of Grid Voltages for a Three-Phase $L C L-$ Type Grid-Connected Inverter, IEEE Transactions on Industrial Electronics, Vol 60, number 6, pp. 2237 2250, 2013.

[12] B. Liu, Q. Wei, C. Zou and S. Duan: Stability Analysis of LCL-Type Grid-Connected Inverter Under SingleLoop Inverter-Side Current Control With Capacitor Voltage Feedforward, IEEE Transactions on Industrial Informatics, Vol. 14, number 2, pp. 691-702, Feb. 2018.

[13] X. Li, J. Fang, Y. Tang, X. Wu and Y. Geng: Capacitor-Voltage Feedforward With Full Delay Compensation to Improve Weak Grids Adaptability of LCL-Filtered Grid-Connected Converters for Distributed Generation Systems, IEEE Transactions on Power Electronics, Vol 33, number 1, pp. 749-764, 2018.

[14] D. Lumbreras, E. L. Barrios, A. Urtasun, A. Ursua, L. Marroyo and P. Sanchis: On the Stability of Advanced Power Electronic Converters: the Generalized Bode Criterion, IEEE Transactions on Power Electronics. 